\title{
Gauge Boson production at the Tevatron
}

\author{
Cecilia E. Gerber ${ }^{\mathrm{a} *}$

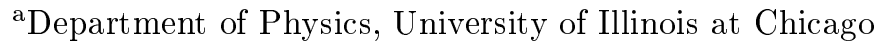 \\ 745 West Taylor St. ChicagoГIL 60607 \\ E-mail: gerber@uic.edu
}

We present measurements on gauge boson production from data taken during 1994-1996 by the DØ and CDF detectors: the differential production cross section of the $W$ boson as a function of the transverse momentum [1,2], the ratio of $W$ and $Z$ differential cross sections [3,4], direct photon cross-sections at $\sqrt{s}=630$ and $1800 \mathrm{GeV}[5,6]$, and studies of Drell-Yan production [7,8]. All measurements are in good agreement with currently available theoretical predictions in most of the measured kinematic range.

\section{The $W$ boson Differential Production Cross Section}

Measurement of the differential cross section for $W$ boson production provides an important test of our understanding of quantum chromodynamics (QCD). Its implications range from impact on the precision determination of the $W$ boson mass to background estimates for new physics phenomena.

When the transverse momentum $\left(p_{T}^{W}\right)$ and the invariant mass $\left(M_{W}\right)$ of the $W$ boson are of the same order $\Gamma$ the production rate can be calculated perturbatively order by order in the strong coupling constant $\alpha_{s}$. For $p_{T}^{W} \ll M_{W} \Gamma$ the calculation is dominated by large logarithms $\approx$ $\alpha_{s} \ln \left(M_{W} / p_{T}^{W}\right)^{2} \Gamma$ which are related to the presence of soft and collinear gluon radiation. Therefore $\Gamma$ at sufficiently small $p_{T}^{W}$ $\Gamma$ fixed-order perturbation theory breaks down and the logarithms must be resummed. The resummation can be carried out in transverse momentum $\left(p_{T}\right)$ space or in impact parameter $(b)$ space via a Fourier transform. Differences between the two formalisms are discussed in Ref. [9].

Although resummation extends the perturbative calculation to lower values of $p_{T}^{W} \Gamma$ a more fundamental barrier is encountered when $p_{T}^{W}$ approaches $\Lambda_{\mathrm{QCD}} \Gamma$ the scale characterizing QCD processes. The strong coupling constant $\alpha_{s}$ becomes large and the perturbative calculation is

\footnotetext{
${ }^{*}$ for the D $\varnothing$ and CDF Collaborations
}

no longer reliable. The problem is circumvented by using a cutoff value and by introducing an additional function that parameterizes the nonperturbative effects [10ח1].

The inclusive differential cross section for $W$ boson production is measured in the electron channel as a function of transverse momentum. We use $85 \mathrm{pb}^{-1}$ of data recorded with the $\mathrm{D} \varnothing$ detector during the 1994-1995 run of the Fermilab Tevatron $p \bar{p}$ collider.

The $W \rightarrow e \nu$ event sample is corrected for kinematic and geometric acceptance $\Gamma$ and detector resolution. The final results for $d \sigma(W \rightarrow$ $e \nu) / d p_{T}^{W}$ are plotted in Fig. $1 \Gamma$ where the data are compared to the combined QCD perturbative and resummed calculation in $b$-space $\Gamma$ computed with published values of the non-perturbative parameters [10]. The error bars on the data points correspond to their statistical uncertainties. The fractional systematic uncertainty is shown as a band in the lower portion of the plot $\Gamma$ and accounts for the errors in the hadronic energy scale and resolution $\Gamma$ the selection efficiency $\Gamma$ and the background. An additional normalization uncertainty of $\pm 4.4 \%$ from the integrated luminosity is not included in any of the plots.

\section{2. $W$ and $Z p_{T}$ Ratio Measurement}

For the analyses of data taken during 19921996 (Fermilab Tevatron Run 1) $\Gamma$ we have used the resummed calculation of Ref. [10] fitted to 

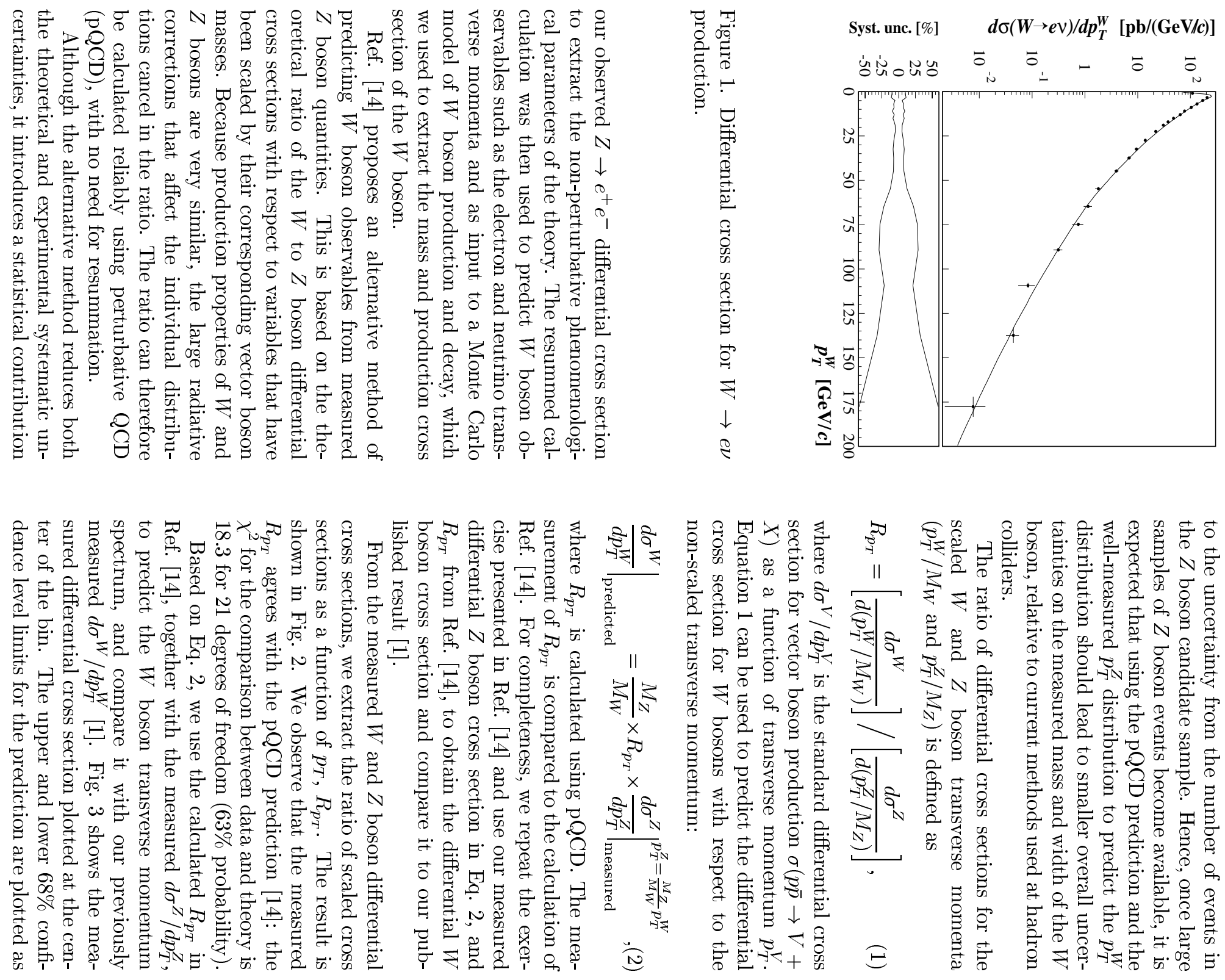
histograms. The extracted transverse momentum distribution agrees well with the measurement.

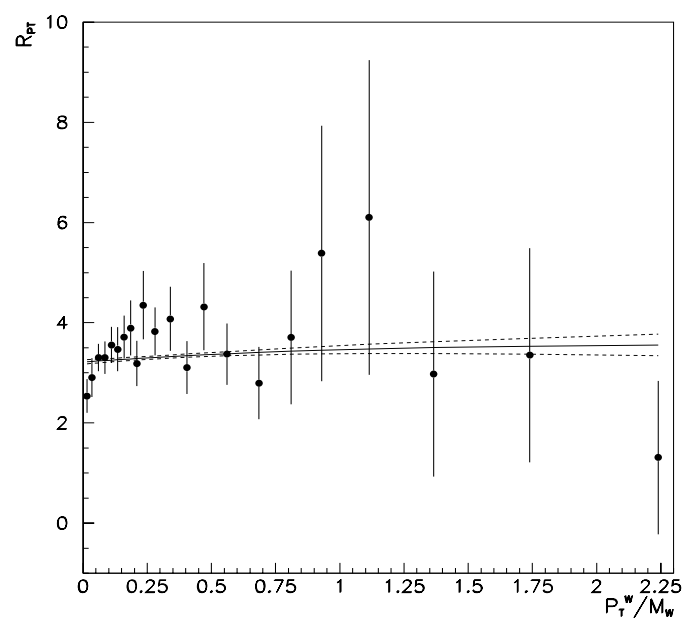

Figure 2. Ratio of scaled differential cross sections $R_{p_{T}}$ for $W$ and $Z$ production.

\section{Direct Photon Cross Sections at $\sqrt{s}=$ 630 and $1800 \mathrm{GeV}$}

Within the framework of Quantum Chromodynamics (QCD) Tisolated single photons are direct photons: produced from the primary partonparton interactions. A measurement of the final state photons provides a probe of QCD without additional complications from fragmentation and jet identification $\Gamma$ providing a powerful and effective means for studying the constituents of hadronic matter.

Previous experiments $\Gamma$ at center-of-mass ener-

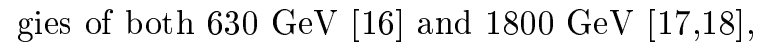
have reported photon production in excess of next-to-leading-order (NLO) QCD predictions at low transverse energies $\left(E_{T}^{\gamma} \sim 30 \mathrm{GeV}\right)$. This disagreement with data could result from gluon radiation not included in NLO calculations or because the parton distributions are not well known.
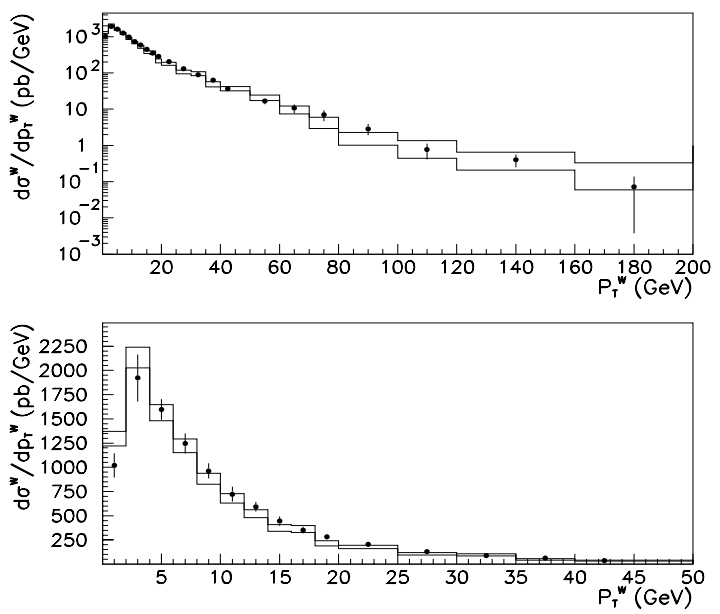

Figure 3. Differential cross section for $W$ boson production as a function of $p_{T}^{W}$.

We present a measurement [5] of the ratio of isolated photon cross sections at different centerof-mass energies $\Gamma 630$ and $1800 \mathrm{GeV}$ by the $\mathrm{D} \varnothing$ experiment. The systematic uncertainty and the sensitivity to the choice of parton distribution functions (PDF) are significantly reduced in the ratio. Photon candidates are required to have calorimeter shower characteristics consistent with that of a single electron $\Gamma$ and must not have a track match in the drift chamber.

In the simple parton model $\Gamma$ the dimensionless cross section $E_{T}^{4} \cdot E \frac{d^{3} \sigma}{d p^{3}} \Gamma$ as a function of $x_{T}=\frac{2 E_{T}}{\sqrt{s}} \Gamma$ is independent of $\sqrt{s}$. Although deviations from such naive scaling are expected $\Gamma$ the dimensionless framework provides a useful context for comparison with QCD. The experimental dimensionless cross section $\Gamma$ averaged over azimuth $\Gamma$ becomes $\sigma_{D}=\frac{E_{T}^{3}}{2 \pi} \cdot d^{2} \sigma / d E_{T} d \eta$. The ratio $\sigma_{D}(\sqrt{s}=630 \mathrm{GeV}) / \sigma_{D}(\sqrt{s}=1800 \mathrm{GeV})$ is determined by combining the cross section reported here with the $\mathrm{D} \varnothing$ measurement at $\sqrt{s}=1800$ $\mathrm{GeV}$ [17]. The ratio is shown as a function of $x_{T}$ in Fig. 4 together with the NLO QCD prediction. 
The probability of agreement from a $\chi^{2}$ test between data and theory is $49 \%(89 \%)$ in the $\mathrm{CC}$ (EC) region. The deviations at low $x_{T}$ are not significant in light of our combined statistical and systematic uncertaintiesTand there exists good agreement between the measured ratio and theory.

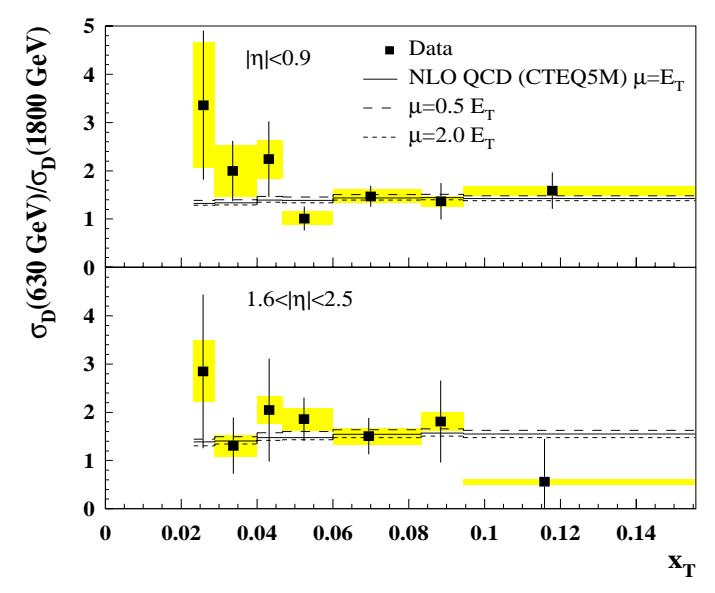

Figure 4 . The ratio of the dimensionless cross sections $\Gamma \sigma_{D}(\sqrt{s}=630 \mathrm{GeV}) / \sigma_{D}(\sqrt{s}=1800 \mathrm{GeV})$ in the central (upper) and forward (lower) rapidity regions.

CDF has measured [6] the cross sections $d^{2} \sigma / d P_{T} d \eta$ for production of isolated direct photons at two center of mass energies (1800 and 630 $\mathrm{GeV}$ ). Figure 5 shows these results as a function of the scaling variable $x_{T}$. One observes a significant disagreement in the ratio that is difficult to explain with conventional theoretical uncertainties such as scale dependence and parton distribution functions. However Tone possibility for the observed discrepancy with NLO QCD is the lack of a complete description of the initial state parton shower in the NLO QCD calculation $\Gamma$ which could give a recoil effect to the photon + jet system
$\left(k_{T}\right)$. To test this hypothesis $\Gamma$ a simplified gaussian smearing was added to the NLO QCD calculation Tgiving the photon+jet system a transverse momentum recoil consistent with that measured in the Drell-Yan process at each center of mass energy. The comparisons of the prediction with the measured ratio are significantly improved with the addition of these amounts of $k_{T}$. Higher order QCD calculations including such effect are becomming available [21] $\Gamma$ but are not ready for detailed comparisons at this time.

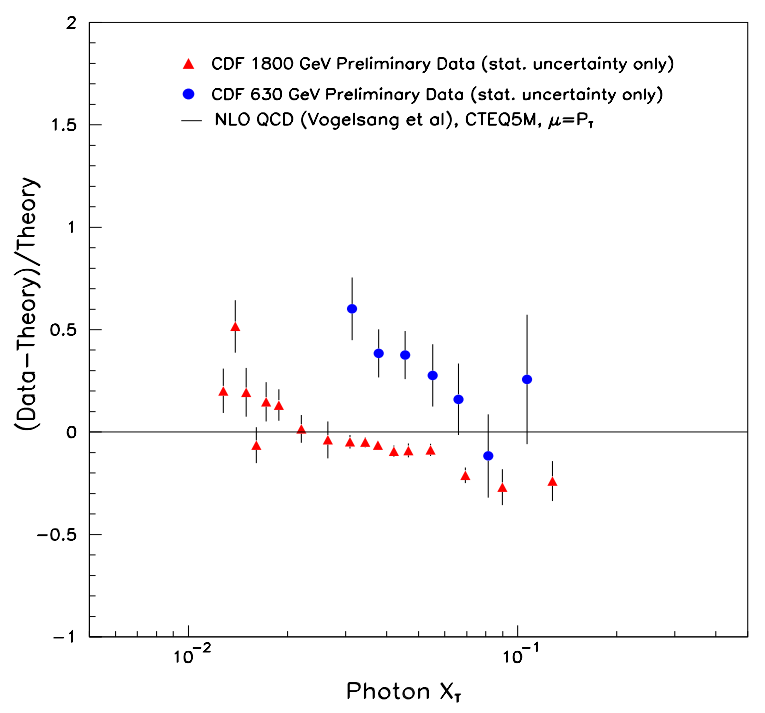

Figure 5. Comparison of 1800 and $630 \mathrm{GeV}$ direct photon data to NLO QCD calculation as a function of photon $x_{T}$.

\section{Studies of high mass Drell-Yan pairs}

We present a new measurement [7] of the mass dependence of the forward-backward asymmetry $\left(A_{F B}\right) \Gamma$ and production cross section $(d \sigma / d M)$ for $e^{+} e^{-}$pairs with mass $M_{e e}>40 \mathrm{GeV}$ from $108 \mathrm{pb}^{-1}$ of data taken by the CDF Collaboration during 1992-1995. The measurements are compared to predictions from the Standard Model 
and from a model with an additional $Z^{\prime}$ gauge boson. Previous measurements by CDF [22] restricted the data to central rapidity leptons (electrons or muons) and measured the $d^{2} \sigma / d M d y$ averaged over central rapidities. This new result includes electrons in the forward calorimeters $\Gamma$ new techniques to reduce the backgrounds from QCD processes $\Gamma$ and reports on measurements of $A_{F B}$ in small bins over a large range in mass (from 40 to $500 \mathrm{GeV}$ ).

Figure 6 compares the measured $d \sigma / d M$ and $A_{F B}$ to theoretical predictions. The upper plot shows $d \sigma / d M$ for $e^{+} e^{-}$pairs for both the CDF and $\mathrm{D} \emptyset$ collaborations $\Gamma$ and $\mu^{+} \mu^{-}$pairs from CDF. The lower plot shows the $A_{F B}$ measurement in the $e^{+} e^{-}$channel from CDF. The Standard Model NNLO prediction is shown as a solid line. The data is in good agreement with the Standard Model predictions. However $\Gamma$ the measured $A_{F B}$ is $2.2 \sigma$ below the Standard Model prediction in the highest mass bin $\Gamma$ from 300 to $600 \mathrm{GeV}$. From the four events in the sampleI three are in the negative hemisphere. A negative asymmetry in this region could result from a new interaction not included in the Standard Model. As one possible example of additional interactions that could be compatible with the measured $A_{F B} \Gamma$ we show in Fig. 6 predictions that include an additional $E_{6} \quad Z^{\prime}$ boson $\Gamma$ with a width of $10 \%$ its mass $\Gamma$ for two masses of $350 \mathrm{GeV}$ and $500 \mathrm{GeV}$ respectively. Although both $\mathrm{CDF}$ and $\mathrm{D} \varnothing$ have set limits on the mass of additional $Z^{\prime}$ bosons of the order of $600 \mathrm{GeV}$ [22I23] those were based on assuming a narrower $Z^{\prime}\left(\Gamma_{Z^{\prime}} \approx 0.01 M_{Z^{\prime}}\right)$ with the same couplings to the three generations than the Standard Model $Z$ boson. Allowing for additional decay modes with larger couplings to third generation reduces the direct limits by 100 to 150 GeV [24]. The high mass Drell-Yan data will be included in global fits to electroweak data to search for physics beyond the Standard Model.

In addition $\Gamma$ we have measured [8] $d \sigma / d y$ over nearly the entire kinematic region of rapidity for $e^{+} e^{-}$pairs in both the Z-boson mass region $\Gamma$ and the high mass mass region of $M_{e e}>116 \mathrm{GeV} / \mathrm{c}^{2}$. Previous measurements were performed in the central rapidity production region $\Gamma$ and a model dependent extrapolation to the forward rapidity region was needed to extract the total cross section for hard processes such as top quark and $W$ or $Z$ boson production. Fig. 7 shows the measured $d \sigma / d y$ distribution for $e^{+} e^{-}$pairs in the $Z$ boson mass window of $66<M_{e e}<116 \mathrm{GeV} / \mathrm{c}^{2}$. The theoretical expectation is consistent with the measurement.
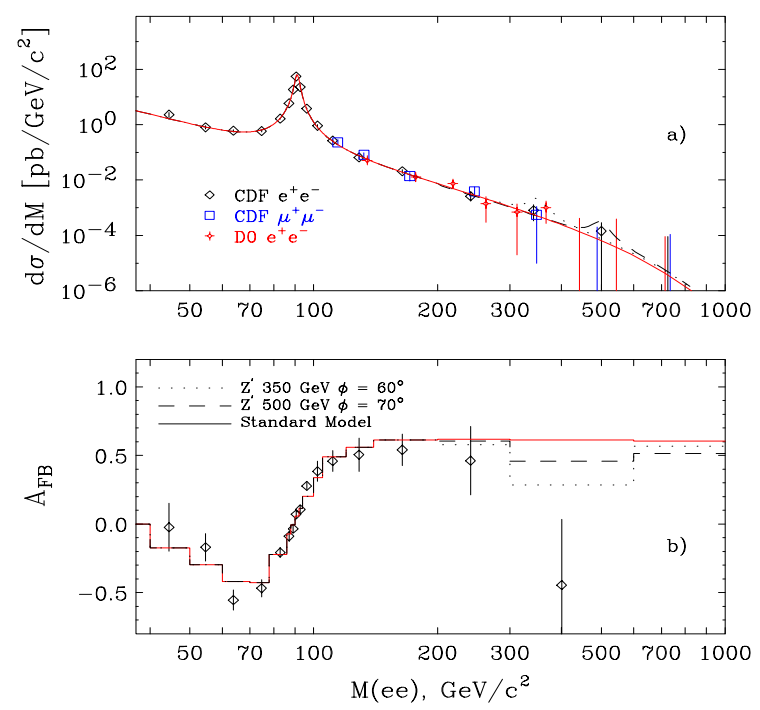

Figure 6. $d \sigma / d M$ (top) and $A_{F B}$ (bottom) distributions from data compared to predictions from the Standard Model (solid line) $\Gamma$ and a model that includes an extra $E_{6} Z^{\prime}$ boson with $M_{Z^{\prime}}=$ $350(500) \mathrm{GeV}$ shown as dotted (dashed) lines.

\section{Summary and Prospects}

Recent results on gauge boson production from D $\varnothing$ and CDF Run 1 data are in good agreement with theoretical predictions. The deviations at low $p_{T}$ are not significant. The study of the $W \mathrm{Z} \Gamma$ and $\gamma$ production mechanisms is important as a direct test of the standard model $\Gamma$ to improve the understanding of the background in top quark and Higgs boson production $\Gamma$ as well as to control the systematics in precision measurements 


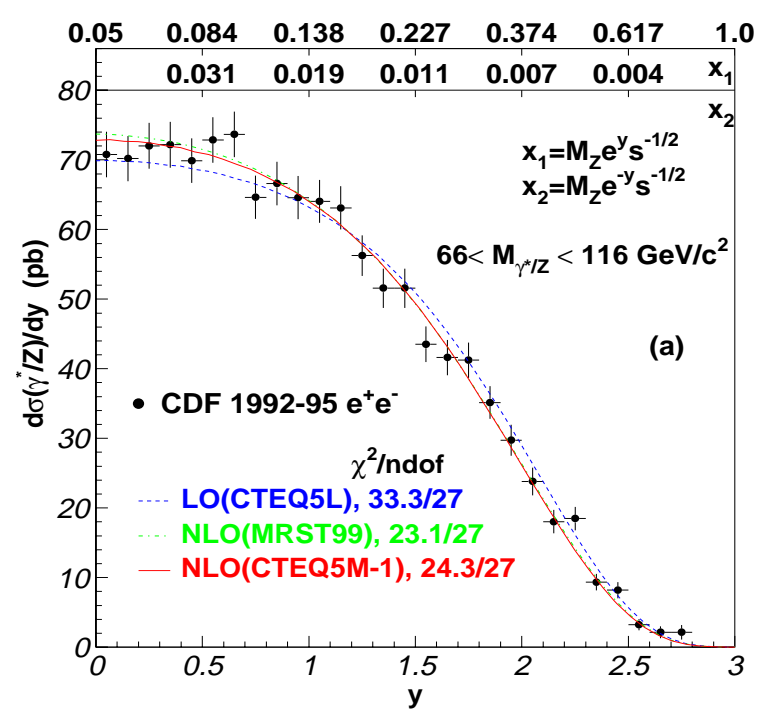

Figure 7. $d \sigma / d y$ for $e^{+} e^{-}$pairs in the $Z$ boson mass window.

such as the $W$ mass. Run 2 has started and we expect $2 \mathrm{fb}^{-} 1$ of data by year 2003 . The much larger number of gauge bosons will allow us to reduce systematics in both the measurements and the predictions. We will also be able to reduce uncertainties in the direct photon cross sections (dominated by the photon purity of the sample) $\Gamma$ as well as to extend the kinematic range of the measurement.

\section{REFERENCES}

1. V. M. Abazov et al. $\Gamma$ (D $\varnothing$ Collaboration) $\Gamma$ Phys. Lett. B 513Г292 (2001).

2. B. Abbott et al. $\Gamma$ (DØ Collaboration)ГPhys. Rev. Lett. 80Г5498 (1998).

3. B. Abbott et al. $(\mathrm{D} \varnothing$ Collaboration)ГPhys. Rev. D 61Г 032004 (2000)Г and Phys. Rev. Lett. 84Г2792 (2000).

4. V. M. Abazov et al.Г (D $\varnothing$ Collaboration) $\Gamma$ Phys. Lett. B 517Г299 (2001).

5. V. M. Abazov et al. $(\mathrm{D} \varnothing$ Collaboration) $\Gamma$ hep-ex/0106026 $\Gamma$ accepted by Phys. Rev. Lett. $\Gamma(2001)$.
6. D. Acosta et al. (CDF Collaboration) Гsubmitted to Phys. Rev. D.

7. T. Affolder et al. (CDF Collaboration) $\Gamma$ Phys. Rev. Lett. 87Г131802 (2001).

8. T. Affolder et al. (CDF Collaboration) ГPhys. Rev. D 631011101 (2000) Rapid Communication

9. P. B. Arnold and R. P. Kauffman TNucl. Phys. B349Г381 (1991).

10. G. A. Ladinsky and C. P. YuanГPhys. Rev. D 50Г4239 (1994).

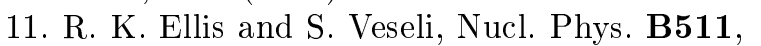
649 (1998).

12. L. Lindemann and G. Zech N Nucl. Instrum. Methods A 354Г516 (1995).

13. G. D. Lafferty and T. R. Wyatt $\Gamma$ Nucl. Instrum. Methods A 355Г541 (1995).

14. W. T. Giele and S. KellerГPhys. Rev. D $57 \Gamma$ 4433 (1998).

15. T. Affolder et al. $\Gamma(\mathrm{CDF}$ Collaboration) ГPhys. Rev. Lett. $84 \Gamma 845$ (2000)

16. J. Alitti et al. (UA2 Collaboration) Г Phys. Lett. B 263Г 544 (1991); R. Ansari et al. (UA2 Collaboration) $\mathrm{Z}$. Phys. C 41Г395 (1988).

17. B. Abbott et al. (DØ Collaboration) Phys. Rev. Lett. 84Г2786 (2000).

18. F. Abe et al. (CDF Collaboration) $\Gamma$ Phys. Rev. Lett. 73Г2662 (1994); F. Abe et al. (CDF Collaboration) $\Gamma$ Phys. Rev. D $48 \Gamma$ 2998 (1993).

19. H. Baer $\Gamma$ J. Ohnemus $\Gamma$ and J.F. Owens $\Gamma$ Phys. Rev. D 42Г 61 (1990); W. Vogelsang and A. Vogt NNucl. Phys. B 453Г334 (1995).

20. CTEQ5MГCTEQ5HJTMRSTГMRSTg $\uparrow$ Гand MRSTg $\downarrow$ were compared. For MRST $\Gamma$ see A.D. Martin et al. Eur. Phys. J. C 14Г 133 (2000).

21. E. Laenen $\Gamma$ G. Sterman $\Gamma$ and W. Vogelsang $\Gamma$ Phys. Rev. Lett. 84Г4296 (2000).

22. F. Abe et al. (CDF Collaboration)ГPhys. Rev. Lett. 79Г2198 (1997) and Phys. Rev. D 59Г52002 (1999).

23. B. Abbott et al. (DØ Collaboration) $\Gamma$ Phys. Rev. Lett. 82Г4769 (2000).

24. A. Bodek and U. BaurThep-ph 0102160. 